\title{
Bioactivity of gentamicin contained in novel transdermal drug delivery systems (TDDS) formulated with biodegradable polyesters
}

Petra Obioma Nnamani ${ }^{1}$, Franklin Chimaobi Kenechukwu ${ }^{1 \star}$, Esther Uju Dibua ${ }^{2}$, Celestine Chidi Ogbonna, 3, ${ }^{2,}$ Mumuni Abdul Momoh ${ }^{1}$, John Dike Nwabueze Ogbonna ${ }^{1}$, David Chibunine Okechukwu ${ }^{1}$, Augustina Uche Olisemeke ${ }^{1}$ and Anthony Amaechi Attama ${ }^{1}$

\author{
${ }^{1}$ Department of Pharmaceutics, University of Nigeria, Nsukka 410001, Enugu State, Nigeria. \\ ${ }^{2}$ Department of Microbiology, University of Nigeria, Nsukka 410001, Enugu State, Nigeria. \\ ${ }^{3}$ Probiotics Research Unit, Microbiology Laboratory, School of Biosciences and Biotechnology, University of Camerino, \\ Via Gentile III da Varano, 62032 Camerino (MC), Italy. \\ ${ }^{4}$ Division of Epidemiology and Prevention, Institute of Human Virology, School of Medicine, University of Maryland, 725 \\ West Lombard Street, Baltimore, MD 21201, USA.
}

Accepted 24 June, 2013

\begin{abstract}
Topical administration of gentamicin, a hydrophilic aminoglycoside antibiotic, is limited by membrane impermeability and toxicity concerns. The purpose of this study was to develop and evaluate the antimicrobial activities of an alternative non-invasive, convenient and cost-effective transdermal drug delivery system (TDDS) containing gentamicin in biodegradable polyester-based matrices. The patches were formulated by solvent evaporation technique using PURASORB ${ }^{\circledR}$ polymers and evaluated for thermal properties, drug content, physicochemical performance, stability, skin irritation on rat skin and antimicrobial activities against five micro-organisms: Staphylococcus aureus, Escherichia coli, Salmonella typhi, Pseudomonas aeruginosa, and Klebsiella pneumoniae. The differential scanning calorimetry (DSC) results indicated compatibilty between the drug and the polymers. In addition, the formulations showed good drug encapsulation, stability, physicochemical properties, tolerability on rabbit skin and higher zones of inhibition compared with a commercially available gentamicin sulphate cream against $S$. aureus, $E$. coli, $S$. typhi and $P$. aeruginosa, while $K$. pneumoniae was mildly susceptible. Compared with the rest of the formulations, patches of PURASORB ${ }^{\circledR}$ PL 32 exhibited the best stability, tolerability on rat skin and bioactivity. This study has shown that transdermal patches of PURASORB $^{\circledR}$ PL 32 represent an alternative delivery system for gentamicin for treatment of infections caused by gentamicin-susceptible micro-organisms.
\end{abstract}

Key words: Antimicrobial activities, bioadhesive strength, gentamicin, PURASORB ${ }^{\circledR}$ polymers, transdermal patches.

\section{INTRODUCTION}

Transdermal drug delivery system is being extensively investigated as a viable alternative to drug delivery with improved bioavailability. Transdermal drug administration generally refers to topical application of agents to healthy 
intact skin either for localized treatment of tissues underlying the skin or for systemic therapy (Valenta and Auner, 2004). It offers many advantages over conventional administration such as enhanced efficacy, increased safety, and greater convenience and improved patient compliance (Valenta and Auner, 2004; Dnyanesh and Vavia, 2003; Chandak and Verma, 2008). Transdermal route permits the use of a relatively potent drug with minimal risk of system toxicity and avoids gastrointestinal degradation and hepatic first-pass metabolism (Mundargi et al., 2007; Mutalik and Udupa, 2004). In case of toxicity, the transdermal patch can easily be removed by the patient (Chang et al., 2006).

Gentamicin sulphate is an aminoglycoside antibiotic commonly used topically in the control of severe Gram positive and Gram negative microbial infections especially in burns and wounds as well as for treating bone and soft tissue infections (Nishijima and Kurokawa, 2002). Despite its benefits, bacterial barriers and adverse effects such as nephrotoxicity, ototoxicity and neurotoxicity upon prolonged use, limit gentamicin daily dosage (Drusano et al., 2007). In fact, many clinicians are reluctant to use it, even for a short term (Singh et al., 2003). Efforts have been made to determine its optimal therapeutic regimens in order to increase its overall efficacy while minimizing drug toxicity. These include various formulation techniques such as formulations for topical administration.

Though topical administration of gentamicin is not painful, the patient may not apply the ointment/cream as often as required. Therefore, there is a need for the preparation of a new dosage form of gentamicin. Owing to the advantages offered by transdermal drug delivery systems (TDDS) over conventional routes of administration (Mundargi et al., 2007; Mutalik and Udupa, 2004), this study was designed to evaluate the transdermal delivery system of gentamicin so as to develop nonparenteral and needle-less (non-invasive) gentamicin preparation that will not only reduce to the barest minimum the systemic toxicity associated with parenteral administration of gentamicin but also ensure patient's compliance. A transdermal patch is not painful and is cost effective (Chang et al., 2006). Technically, the patch is placed in a part of the body which releases the drug into the body for a long period of time (Valenta and Auner, 2004; Dnyanesh and Vavia, 2003; Chandak and Verma, 2008). Polymeric matrices are usually employed as carriers for transdermal delivery of drugs/actives (Dnyanesh and Vavia, 2003; Chandak and Verma, 2008; Mundargi et al., 2007; Mutalik and Udupa, 2004; Verma and lyker, 2002; Gupta and Mukherjee, 2003; Lyman, 2007). The novelty embodied in this study lies in the formulation of gentamicin transdermal patches using PURASORB ${ }^{\circledR}$ polymers, a well-established, safe, biocompatible and resorbable excipients commonly employed in the formulation of controlled release drug delivery systems. These biodegradable polyesters have wide applications, including as orthopedic implant devices, surgical sutures, cardiovascular products, tissue regeneration scaffolds, among others. PURASORB ${ }^{\circledR}$ materials allow for maximum flexibility in formulation technologies, ranging from extrusion and solvent processing to spray drying. Moreover, they are the material of choice for the production of implants, microspheres, and depot systems (Yasukawa et al., 2001; Avitable et al., 2001; Arora and Mukherjee, 2002).

The objective of this study, therefore, was to design and formulate transdermal patches incorporating gentamicin using biodegradable polyesters for the purpose of enhancing the delivery of the drug, by providing controlled delivery of the drug. The suitability of four different biodegradable polyesters (PURASORB ${ }^{\circledR}$ polymers: PLGA, PDL 05, PL 32 and PDL 04) for this purpose was assessed by evaluating some of the physicochemical properties of the patches formed, the efficiency of incorporation of the drug in the patches as well as the bioactivity of the incorporated drug.

\section{MATERIALS AND METHODS}

The following materials were used without further purification: Gentamicin (Schering, Rockville, MD, USA), Poly(D, L-lactide-coglycolide PLGA)-PURASORB ${ }^{\circledR}$ PDLG 7502A, poly(L-lactide) PURASORB $\mathrm{PL}^{\circledR}$ 32, poly(DL-lactide)-PURASORB ${ }^{\circledR}$ PDL 04, and poly(DL-lactide) -PURASORB ${ }^{\circledR}$ PDL 05 (PURAC biochem by Gorinchem, Holland), ethyl acetate, sodium borate, ophthalidialdehyde and 2-mercaptoethanol (Sigma-Aldrich, USA), polyvinyl alcohol, propylene glycol (Merck, Germany), isopropanol, methanol and formalin (Adwic El-Nasr, Chemical Co., Cairo, Egypt), sodium hydroxide (BDH, England) and distilled water (Lion water, UNN, Nigeria). Clinical isolates of Staphylococcus aureus ATCC 13703, Salmonella typhi ATCC 786 and Escherichia coli ATCC 9637 were obtained from Bishop Shanahan Hospital, Nsukka, Nigeria; whereas laboratory isolates of Pseudomonas aeruginosa and Klebsiella pneumoniae were procured from the Microbiology Laboratory, Department of Pharmaceutics of our University. Gentamicin sulphate cream USP, $0.1 \%$ (Perrigo Bronx, New York, USA) was used as a commercially available topical gentamicin cream. All other laboratory materials were of analytical grade. All experiments involving the use of animals were conducted in accordance with Ethical Guidelines of Animal Care and Use Committee (Research Ethics Committee) of University of Nigeria, Nsukka, following the $18^{\text {th }}$ WMA General Assembly Helsinki, June 1964 and updated by the $59^{\text {th }}$ WMA General Assembly, Seoul, October 2008.

\section{Preparation of transdermal patches}

The patches were prepared by solvent evaporation technique (Amunuaikit et al., 2005), using gentamicin, plasticizers and other film forming polymers. Gentamicin $(5 \mathrm{~g})$ was dissolved with distilled water $(5 \mathrm{ml})$ in a beaker followed by addition of $10 \mathrm{~g}$ of propylene glycol. The mixture was stirred continuously until a solution (drug reservior) was formed. The backing membrane was cast by weighing approximately $10 \mathrm{~g}$ of the film forming polymer (PLGA) into a separate beaker, adding about $70 \mathrm{ml}$ of ethyl acetate and 
Table 1. Composition of transdermal patches.

\begin{tabular}{lcccc}
\hline \multirow{2}{*}{ Ingredient } & \multicolumn{4}{c}{ Formulation code } \\
\cline { 2 - 5 } & PLGA & PDL 05 & PL 32 & PDL 04 \\
\hline Drug $(\mathrm{g})$ & 5 & 5 & 5 & 5 \\
Polymer $(\mathrm{g})$ & 10 & 10 & 10 & 10 \\
Propylene glycol $(\mathrm{g})$ & 10 & 10 & 10 & 10 \\
Water $(\mathrm{ml})$ & 5 & 5 & 5 & 5 \\
Chloroform $(\mathrm{ml})$ & $\ldots$ & $\ldots$ & 180 & $\ldots$ \\
Ethyl acetate $(\mathrm{ml})$ & 70 & 63 & $\ldots$ & 5 \\
$0.1 \mathrm{~N} \mathrm{NaOH}(\mathrm{ml})$ & $\ldots$ & $\ldots$ & 5 & 5 \\
\hline
\end{tabular}

votexing (Vortex Genie Bouxemia. N.Y 11716, USA) the mixture for $5 \mathrm{~min}$. Subsequently, the drug-contaning solution (drug reservior) was then poured into the solution containing the PLGA (backing membrane). This dispersion was properly stirred and poured into an aluminium foil-lined petri dish of defined area $\left(10 \mathrm{~cm}^{2}\right)$. A funnel of suitable size was inverted over the petri dish to minimize solvent evaporation. Casting solvent was then allowed to evaporate $48 \mathrm{~h}$ to obtain dry films. The above procedure was repeated using PDL 05, PL 32 and PDL 04 as the film forming polymers as well as appropriate quantities of either or both ethylacetate, chloroform and sodium hydroxide $(0.1 \mathrm{~N} \mathrm{NaOH})$, as depicted in Table 1. The patches were stored between sheets of wax paper in a desiccator until further analysis.

\section{Differential scanning calorimetry (DSC)}

The stability and compatibility of gentamicin and different polymers to be used for the development of gentamicin transdermal film formulations was studied using a differential scanning calorimeter (Netzsch DSC 204 F1, Germany). Sample (2.5 - $5 \mathrm{mg}$ ), placed in an aluminum crucible cell was firmly crimped with the lid to provide an adequate seal. The thermal properties such as melting temperature, enthalpy and glass transition of the drug and transdermal patches were deteremined in the range of $35-190^{\circ} \mathrm{C}$ under a $20 \mathrm{ml} / \mathrm{min}$ nitrogen flux at a heating rate of $10^{\circ} \mathrm{C} / \mathrm{min}$. The baselines were determined using an empty pan, and all the thermograms were baseline corrected.

\section{Characterization of gentamicin transdermal films}

The patches were characterized according to standard procedures with respect to physical appearance, thickness (Devi et al., 2003), weight variation (Gupta and Mukherjee, 2003), moisture content (Chang et al, 2006), moisture uptake (Lyman, 2007), film folding endurance (Zhang et al., 1994), drug content (Chang et al., 2006; Bazigha et al., 2011), in vitro bioadhesive strength (Ganesh et al., 2011; Verma and Chandak, 2009) and skin irritancy (National Committe for Clinical Laboratory Standards, 2003).

\section{Bioevaluation of transdemal patches}

The antimicrobial activity of the transdermal patches was tested against each isolates using the agar diffusion technique (Umeyor et al., 2011). This method depends on the diffusion of antibiotics from holes on the surface of the microbial seeded agar. This test was carried out for the prepared gentamicin patches as well as the commercially available gentamicin sulphate cream. Molten nutrient agar $(20 \mathrm{ml})$ was inoculated with $0.1 \mathrm{ml}$ of $S$. aureus broth culture. It was mixed thoroughly, poured into sterile Petri dishes and rotated for even distribution of the organism. The agar plates were allowed to set and a sterile cork borer ( $8 \mathrm{~mm}$ diameter) was used to bore holes in the seeded agar medium. For the tested patch formulae as well as the commercially available cream, a definite quantity containing equivalent amount of gentamicin was accurately weighed and inserted in a corresponding hole. The plates were allowed to stand at room temperature for $15 \mathrm{~min}$ to enable prediffusion before incubating at $37 \pm 0.5^{\circ} \mathrm{C}$ for $24 \mathrm{~h}$. The experiment was repeated for E. coli, $P$. aeruginosa, S. typhi and $K$. pneumoniae. Three replicate tests were performed in each case. Growth was examined after incubation and the diameter of each inhibition zone was measured and the average determined.

\section{Stability study of transdermal patches}

Time resolved stability studies were carried out on the various batches of the formulations following the $\mathrm{ICH}$ guidelines (Verma and Chandak, 2009). The transdermal patches were stored at $40 \pm$ $0.5^{\circ} \mathrm{C}$ in a humidity chamber having a $\mathrm{RH}$ of $75 \pm 5 \%$. After four weeks, six months and one year of storage, the patches were withdrawn and evaluated for the drug content following the method stated above.

\section{Statistical analysis}

All experiments were performed in replicates for validity of statistical analysis. Results were expressed as mean \pm SD. ANOVA and Student's t-test were performed on the data sets generated using Statistical Package for the Social Sciences (SPSS). Differences were considered significant for $p$-values $<0.05$.

\section{RESULTS AND DISCUSSION}

\section{Differential scanning calorimetry (DSC)}

Table 2 presents the thermal properties of the transdermal patches. The physicochemical compatibility of the drug and the polymers studied by differential scanning calorimetry suggested absence of any incompatibility. The results revealed the compatibility of gentamicin and the polymers as well as the stability of the drug in the polymeric matrices (formulations). This is because the formulations gave lower endotherms than gentamicin, 
Table 2. Thermal properties of gentamicin transdermal patches.

\begin{tabular}{|c|c|c|c|c|c|}
\hline \multirow{3}{*}{ Formulation code } & \multicolumn{5}{|c|}{ Thermal properties } \\
\hline & \multirow{2}{*}{$\begin{array}{l}\text { Melting point } \\
\left({ }^{\circ} \mathrm{C}\right)\end{array}$} & \multirow{2}{*}{$\begin{array}{l}\text { Enthalpy } \\
\text { (Mw/mg) }\end{array}$} & \multicolumn{3}{|c|}{ Glass transition temperature $\left(\mathrm{T}_{\mathrm{g}}\right)\left({ }^{\circ} \mathrm{C}\right)$} \\
\hline & & & Onset & Middle & End \\
\hline PLGA & - & - & 31.3 & 26.9 & 22.4 \\
\hline PDL 05 & - & - & 38.7 & 40.1 & 41.5 \\
\hline PL 32 & 180.7 & -1.981 & 80.2 & 89.0 & 97.8 \\
\hline PDL 04 & 126.5 & -5.155 & 39.9 & 42.9 & 46.0 \\
\hline
\end{tabular}

PL 32, PDL 04, PDL 05 and PLGA are gentamicin-loaded patches containing PURASORB ${ }^{\circledR}$ PL 32, PDL 04, PDL 05 and PLGA, respectively.

Table 3. Properties of gentamicin transdermal patches

\begin{tabular}{lcccc}
\hline \multirow{2}{*}{ Parameter } & \multicolumn{4}{c}{ Formulation code } \\
\cline { 2 - 5 } & PLGA $^{\mathbf{a}, \mathbf{b}}$ & PDL 05 $^{\mathbf{a , b}}$ & PL 32 $^{\mathbf{a , b}}$ & PDL 04 $^{\mathbf{a}, \mathbf{b}}$ \\
\hline Thickness $(\mu \mathrm{m})$ & $390.50 \pm 9.08$ & $400.60 \pm 2.18$ & $384.20 \pm 5.97$ & $405.80 \pm 6.03$ \\
Weight variation $(\mathrm{mg})$ & $20.90 \pm 0.27$ & $21.30 \pm 0.25$ & $20.70 \pm 0.01$ & $21.60 \pm 0.15$ \\
Drug content (\%) & $97.60 \pm 2.43$ & $95.80 \pm 1.49$ & $98.90 \pm 2.45$ & $96.80 \pm 2.42$ \\
Folding endurance & $316.50 \pm 4.17$ & $327.10 \pm 3.89$ & $307.90 \pm 5.02$ & $311.40 \pm 8.09$ \\
Tensile strength (dyne cm ${ }^{-2}$ ) & $75.60 \pm 1.07$ & $68.70 \pm 1.04$ & $81.40 \pm 2.03$ & $60.50 \pm 1.40$ \\
Moisture absorption $(\%)$ & $2.70 \pm 0.28$ & $2.90 \pm 0.67$ & $1.90 \pm 0.33$ & $2.60 \pm 0.20$ \\
Moisture content (\%) & $1.60 \pm 0.29$ & $1.20 \pm 0.07$ & $1.50 \pm 0.14$ & $1.70 \pm 0.38$ \\
\hline
\end{tabular}

${ }^{a}$ Mean $\pm S D,{ }^{b} n=3, P L 32$, PDL 04, PDL 05 and PLGA are gentamicin-loaded patches containing PURASORB ${ }^{\circledR}$ PL 32, PDL 04, PDL 05 and PLGA, respectively.

implying that gentamicin exists in amorphous state in the formulations and also is properly solubilized in the matrix systems (Nnamani et al., 2010). Moreover, there was general disappearance of drug peak in all patches but PURASORB ${ }^{\circledR}$ PL 32 patch had the least enthalpy $(-1.981$ $\mathrm{mW} / \mathrm{mg}$ ) reflecting the degree of disorder in the matrix.

\section{Characterization of gentamicin transdermal films}

The results of the physicochemical characterization of the patches are shown in Table 3. All the patches of the different polymers were transparent, colourless, smooth and uniform but the PDL 05 polymer had the greatest clarity. The results indicated that the formulated gentamicin-loaded patches exhibited good organoleptic and physicochemical properties (Valenta and Auner, 2004; Dnynesh and Vavia, 2003; Chandak and Verma, 2008; Mundargi et al., 2007; Mutalik and Udupa, 2004).

\section{Bioevaluation of transdemal patches}

The results of drug release studies using the agar plate diffusion method are presented in Figure 1. Compared with the commercially available gentamicin sulphate cream, all batches of the transdermal patches gave great zone of inhibition against $S$. aureus, E. coli, S. typhi and $P$. aeruginosa, while $K$. pneumoniae was mildly susceptible to the formulations. From the results presented in Figure 1, the patch formulations gave these zones of inhibition in decreasing order of magnitude: $\mathrm{PL}$ 32>PLGA $>\mathrm{PDL}$ 04>PDL 05 against E. coli, $\mathrm{PL} 32>\mathrm{PDL}$ 05>PLGA $>$ PDL 04 against $S$. typhi, PL 32>PDL 04>PLGA $>$ PDL 05 against $S$. aureus, PL 32>PDL 05>PDL 04>PLGA against $K$. pneumonia, and PL 32> PDL $04>$ PDL $05>$ PLGA against $P$. aeruginosa. Compared with the rest of the batches of the formulations, PURASORB ${ }^{\circledR}$ PL 32 gave the greatest zone of inhibition against all the transdermal patches employed in the study. While PURASORB ${ }^{\circledR}$ PDL 04 gave the least zone of inhibition against $S$. typhi, PURASORB ${ }^{\circledR}$ PDL 05 showed the least inhibition zone diameter against $S$. aureus and $E$. coli whereas PLGA exhibited the smallest zone of inhibition against $K$. pneumoniae and $P$. aeruginosa, respectively.

The microbiological test was performed to establish that gentamicin sulphate did not lose activity during formulation and after short-term storage, and was done one month after preparation. The determination of inhibition zone diameter (IZD) using agar plate method was based on the diffusion of an antibiotic agent or 


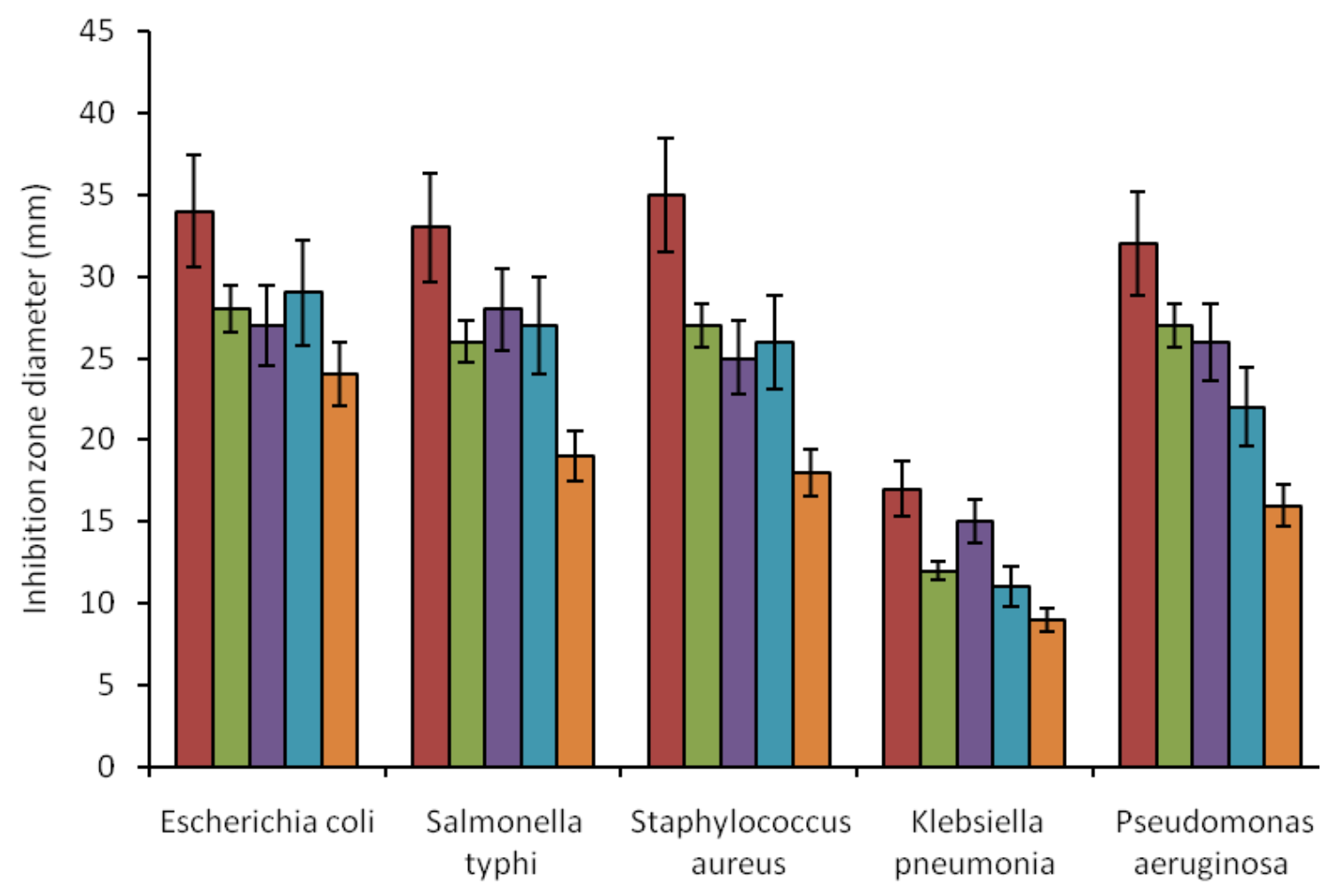

Bacterial isolates

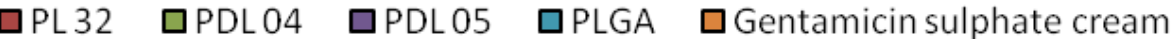

Figure 1. Gentamicin release from drug-loaded transdermal patches as a function of inhibition zone diameter using the bacterial isolates obtained after 1 month of storage $(n=3)$. PL 32, PDL 04, PDL 05 and PLGA are gentamicin-loaded patches containing PURASORB ${ }^{\circledR}$ PL 32, PDL 04, PDL 05 and PLGA, respectively.

formulation thereof through a solidified nutrient agar (Umeyor et al., 2011). From the results presented in Figure 1, the transdermal patches released gentamicin which showed higher IZD compared with the commercially available gentamicin sulphate cream against $E$. coli, S. typhi, S. aureus and $P$. aeruginosa, while $K$. pneumoniae was mildly susceptible to the formulations. Patches of PURASORB ${ }^{\circledR}$ PL 32 showed the best bioactivity. The results indicate that gentamicin loaded into transdermal patches produced very significant zones of inhibition against the Gram positive organism ( $S$. aureus) and Gram negative organisms (S. typhi, E. coli, $S$. typhi, and $P$. aeruginosa) used in the study. The release of gentamicin from the patches depended on the type of polymer since the results showed that batches formulated with PURASORB ${ }^{\circledR}$ PL 32 gave the greatest zones of inhibition against most of the isolates compared with the rest of the formulations. It is discernible from Figure 1 that the biodegradable polyesters, especially PURASORB ${ }^{\circledR} P L 32$, enhanced the release of gentamicin and therefore are good carrier for its transdermal delivery (Valenta and Auner, 2004).

\section{Stability study of transdermal patches}

Figure 2 shows the drug content of the formulations after storage for one year. It is always very important to assess the stability of novel formulations. Stability could be viewed from the degradation of the active ingredients or physical property of the formulation (Panigrahi et al., 2005; Umeyor et al., 2012). In order to determine the change in drug content on storage, stability study was carried out. The results of the stability studies showed that the content of gentamicin in the formulations was not significantly changed on storage, as is evident from Figure 2. The result indicates that the formulations were stable on the required storage condition. In other words, there was a non significant change in the content of gentamicin in the formulations based on the required 


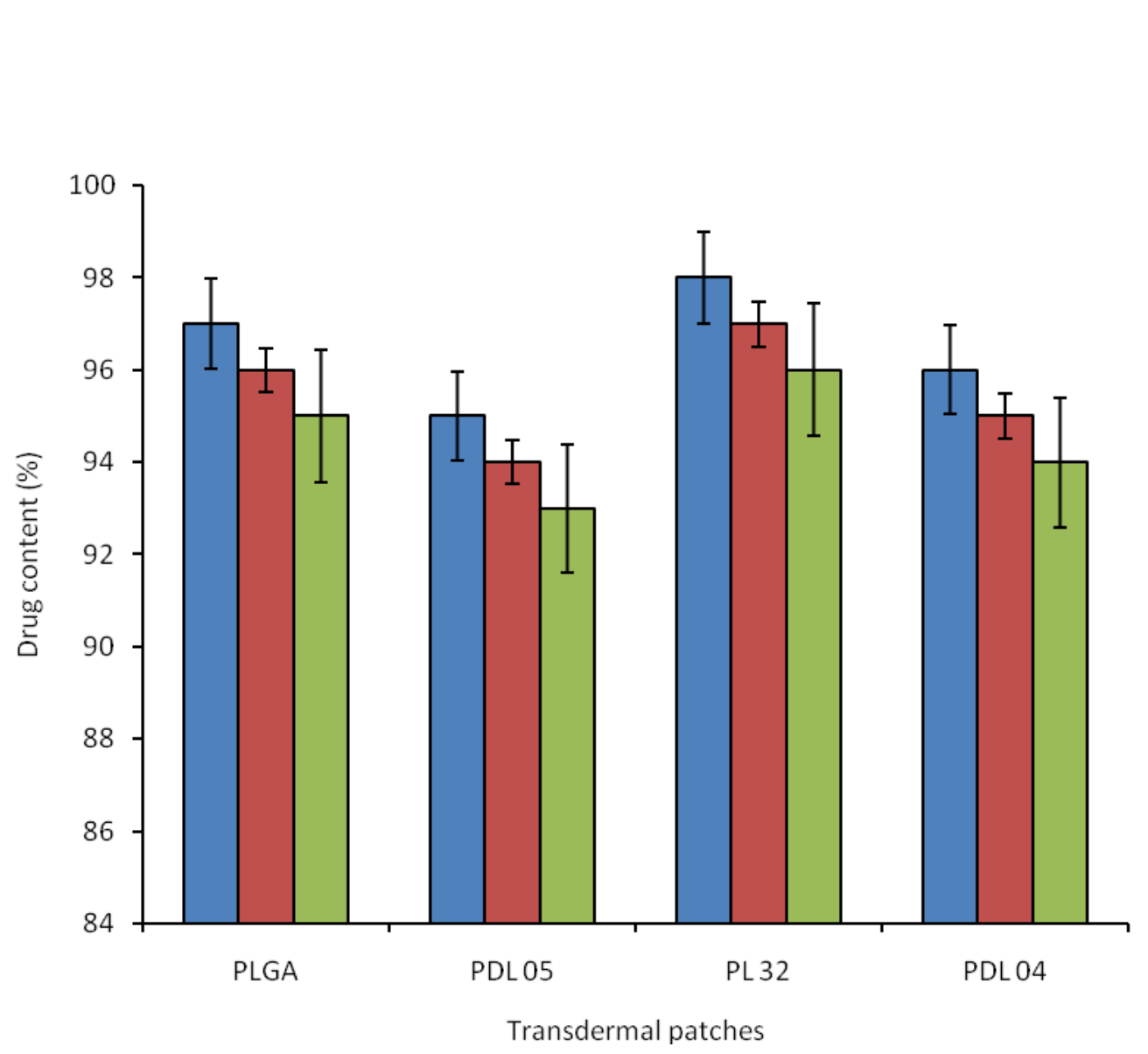

Figure 2. Content of gentamicin in the transdermal patches after storage for one year $(n=3)$. PL 32, PDL 04, PDL 05 and PLGA are gentamicin-loaded patches containing PURASORB ${ }^{\circledR}$ PL 32, PDL 04, PDL 05 and PLGA, respectively

storage conditions.

\section{Conclusion}

Polymeric and/or lipid carriers could be exploited to improve the delivery of therapeutic molecules. In this study, gentamicin-loaded transdermal patches based on biodegradable polyesters were successfully prepared by the solvent evaporation technique, which is simple, cheap and reproducible. All the formulations showed good stability, tolerability on rat skin and physicochemical properties. The isolates of $E$. coli, $S$. aureus, $P$. aeruginosa, S. typhi and $K$. pneumonia were susceptible to gentamicin incorporated into the transdermal patches. In other words, the antibacterial properties of the drug was retained, with PURASORB ${ }^{\circledR}$ PL 32 patches exhibiting the best bioactivity compared with the commercially available gentamicin sulphate cream that gave the least. It follows that this delivery system (PURASORB ${ }^{\circledR}$ PL 32 patches) could offer a better and more promising approach for the treatment of topical infections caused by gentamicin-susceptible micro-organisms than the commercially available topical gentamicin sulphate cream.

\section{ACKNOWLEDGEMENTS}

We wish to thank PURAC Biochem, Gorinchem, Holland for providing samples of the polyesters (PURASORB ${ }^{\circledR} \mathrm{PL}$ 32, PDL 04, PDL 05 and PLGA) used in the study.

\section{ABBREVIATIONS}

TDDS, transermal drug delivery systems; PL 32, PDL 04, PDL 05 and PLGA are gentamicin-loaded patches containing PURASORB ${ }^{\circledR}$ PL 32, PDL 04, PDL 05 and PLGA respectively; APIs, active pharmaceutical ingredients; IZD, inhibition zone diameter; DSC, differential scanning calorimetry. 


\section{REFERENCES}

Amunuaikit C, Ikeuchi I, Ogawara K, Higaki K, Kimura T (2005). Skin permeation of propanolol from polymeric film containing terpene enhancers for transdermal use. Int. J. Pharm. 289:167-178.

Arora P, Mukherjee P (2002). Design, development, physicochemical, and in vitro and in vivo evaluation of transdermal patches containing diclofenac diethylammonium salt. J. Pharm. Sci. 91:2076-2089.

Avitable T, Marano F, Castiglione F, Bucolo C, Cro M, Ambrosio L (2001). Biocompatibility and biodegradation of intravitreal hyaluronan implants in rabbits. Biomaterials 22:195-200.

Bazigha KAR, Uday SA, Omar S, Alaa AAR (2011). Design and evaluation of a bioadhesive film for transdermal delivery of propranolol hydrochloride. Acta Pharm. 61:271-287.

Chandak AR, Verma PRP (2008). Development and evaluation of HPMC based matrices for transdermal patches of tramadol. Clin Res. Reg. Affairs 25:13-30.

Chang HI, Perrie Y, Coombes AGA (2006). Delivery of the antibiotic gentamicin sulphate from precipitation cast matrices of polycaprolactone. J. Control. Rel. 110:414-421.

Devi VK, Saisivam S, Maria GR, Deepti PU (2003). Design and evaluation of matrix diffusion controlled transdermal patches of verapamil hydrochloride. Drug Dev. Ind. Pharm. 29:495-503.

Dnyanesh NT, Vavia PR (2003). Acrylate-based transdermal therapeutic system of nitrendipine. Drug Dev. Ind. Pharm. 29:71-78.

Drusano GL, Ambrose PG, Bhavnani SM, Bertino JS, Nafziger AN, Louie A (2007). Back to the future: using aminoglycosides again and how to dose them optimally. Clin. Infect. Dis. 45:755-760.

Ganesh R, Falguni M, Jayvadan P (2011). Formulation and evaluation of mucoadhesive glipizide films. Acta Pharm. 61:203-216.

Gupta R, Mukherjee B (2003). Development and in vitro evaluation of diltiazem hydrochloride transdermal patches based on povidone-ethyl cellulose matrices. Drug Dev. Ind. Pharm. 29:1-7.

Lyman DJ (2007). Biomedical materials. In: Pramein EN (Ed.), Encyclopedia of Polymer Science and Technology, Pharmaceutical Press, London, pp.1-19.

Mundargi RC, Patil SA, Agnihotri SA, Aminabhavi TM (2007). Evaluation and controlled release characteristics of modified xanthan films for transdermal delivery of atenolol. Drug Dev. Ind. Pharm. 33:79-90.

Mutalik S, Udupa N (2004). Glibenclamide transdermal patches: Physicochemical, pharmacodynamic and pharmacokinetic evaluations. J. Pharm. Sci. 93:1577-1594.

National Committee for Clinical Laboratory Standards (2003). Methods for dilution antimicrobial susceptibility tests for bacteria that grow aerobically. Approved standard, Sixth Edition, Wayne, PA7 NCCLS, 2003.
Nishijima S, Kurokawa I (2002). Antimicrobial resistance of Staphylococcus aureus isolated from skin infections. Int. J. Antimicrob. Agents 19:241-243.

Nnamani PO, Attama AA, Ibezim EC, Adikwu MU (2010). SRMS $142-$ based solid lipid microparticles: Application in oral delivery of glibenclamide to diabetic rats. Eur. J. Pharm. Biopharm. 76:68-74.

Panigrahi L, Pattnaik S, Ghosal SK (2005). The effect of pH and organic ester penetration enhancers on skin permeation kinetics of terbutaline sulfate from pseudolatex-type transdermal delivery systems through mouse and human cadaver skins. AAPS Pharm. Sci. Tech. 6:167-173.

Singh J, Tripathi KT, Sakia TR (2003). Effect of penetration enhancers on the in vitro transport of ephedrine through rat skin and human epidermis from matrix based transdermal formulations. Drug Dev. Ind. Pharm. 19:1623-1628.

Umeyor CE, Kenechukwu FC, Ogbonna JDN, Builders PF, Attama AA (2011). Preliminary studies on the functional properties of gentamicin in SRMS-based solid lipid microparticles. Int. J. Novel Drug Deliv. Tech. 1: 130-142.

Umeyor CE, Kenechukwu FC, Ogbonna JDN, Chime SA, Attama AA (2012). Preparation of novel solid lipid microparticles loaded with gentamicin and its evaluation in vitro and in vivo. J. Microencapsul. 29: 296-307.

Valenta C, Auner BG (2004). The use of polymers for dermal and transdermal delivery. Eur. J. Pharm. Biopharm. 58: 279-289.

Verma PRP, Chandak AR (2009). Development of matrix controlled transdermal delivery systems of pentazocine: in vitrolin vivo performance. Acta Pharm. 59:171-186.

Verma PRP, lyker SS (2002). Transdermal delivery of propronolol using mixed grades of Eudragit: design and in vitro and in vivo evaluation. Drug Dev. Ind. Pharm. 25:1246-1251.

Yasukawa MDT, Kimura H, Tabata Y, Ogura Y (2001). Biodegradable scleral plugs for vitreo-retinal drug delivery. Adv. Drug Del. Rev. 52:25-36.

Zhang X, Wyss UP, Pichora D, Goosen MFA (1994). Biodegradable controlled antibiotic release devices for osteomyelitis: optimization of release properties. J. Pharm. Pharmacol. 46:718-724. 